\title{
BLUMBERG PROPERTY VERSUS ALMOST CONTINUITY
}

\section{Z. PIOTROWSKI}

\author{
Department of Mathematical \\ and Computer Sciences \\ Youngstown State University \\ Youngstown, OH 44555 U.S.A.
}

(Received May 21, 1986)

\begin{abstract}
Some relations between the Blumberg property and almost continuity are
\end{abstract} established. A problem of S.-Y. LIn and Y.-F. Lin is completely resolved.

KEY WORDS AND PHRASES. Blumberg property, almost continuity, Baire space 1980 MATHEMATICS SUBJECT CLASSIFICATION CODE. Primary 54C10; Secondary $54 \mathrm{C} 30$

1. INTRODUCTION A function $\mathrm{f}: \mathrm{X}+\mathrm{Y}$ is almost continuous if for every $\mathrm{x} \varepsilon \mathrm{X}$ and for each open set $V \subset Y$ containing $f(x), C l\left(f^{-1}(V)\right)$ is a neighborhood of $x$.

In Theorem 1 p. 453, S. $-Y . T$. Lin [1] proved the following.

THEOREM A. If $\mathrm{f}: \mathrm{X} \rightarrow \mathrm{Y}$ is a function from a Bafre space $X$ to a second countable space $Y$, then the function $f$ is almost continuous on a dense subset of $X$.

Next, Lin and Lin[2] Theorem 3 p. 184, the following characterization of Baire spaces is given, see also Tong [3] and Tong [4].

THEOREM B. Let $Y$ be an arbitrary second countable, infinite Hausdorff space. Then a space $X$ is Baire iff every function $f: X \rightarrow Y$ is almost continuous on a dense subset of $X$.

Also, Lin and Lin [2] 53. Open problems, Problem 1 p. 185 the following question was asked. Do Theorems $A$ and $B$ remain true without assuming second countability on the range space $Y$ ?

Obviously, one cannot go as far as to have $Y$ first countable, for otherwise the Identity function from the reals with the usual topology into the reals with the discrete topology is a counterexample.

But how far can we go? Can Y have, say, a open-hereditarily countable pseudobase and still satisfy Theorem A (thus B)? Is Theorem A or Theorem B true if $X$ is a $K$-Baire space and $Y$ has weight $K$ ? Suppose every function $f: \mathbb{R} \rightarrow Y$ satisfies the conclusion of Theorem $A$; must $Y$ then have a countable base?

In this paper we shall answer all of these questions.

Let us start by showing that the requirement of Theorems $A$ and $B$ that $Y$ is second countable can not be relaxed to one such that $\mathrm{Y}$ has both a open-hereditarily countable pseudo-base and is hereditarily Lindelöf, see Lutzer [5] for the properties of Sorgenfrey line. 
EXAMPLE 1. Let $X$ be the reals with the usual topology, let $Y$ be the Sorgenfrey line and let $f: X \rightarrow Y$ be the identity function. Not only is $f$ nowhere almost continuous, but the restriction to any dense subset $D$ of $X$ is nowhere almost continuous. In fact, let $x \in X$ be given. Consider the open (in $Y$ ) neighborhood $[f(x), f(x)+1$ ) of $f(x)$. Clearly $C 1_{D}\left(f^{-1}[f(x), f(x)+1)\right.$ ) is not a neighborhood of $x$ (in $\left.D\right)$.

2. A GENERALIZATION. Another target we may look at is a possible analogue of Theorems $A$ and $B$ for arbitrary cardinals. We shall show that Theorem $A$ is transferable, but its converse requires the additional assumption that $\mathrm{Y}$ is metric. Further, Theorem A then holds even in the general case for the following "specialized" class of functions.

Recall that a function $\mathrm{f}: \mathrm{X} \rightarrow \mathrm{Y}$ is categorically almost continuous (or: C-almost continuous), if for every $x_{0} \varepsilon X$ and each open set $V \subset Y, x_{0} \varepsilon f^{-1}(V)$ implies $x_{0} \in$ Int $D\left(f^{-1}(V)\right)$, where $D(S)$ denotes the set of all points $x \in X$ which are of second category relative to $S$, $1 . e ., U \cap S$ is of second category in $X$ for every open $U$ containing $x$.

Clearly, every categorically almost continuous functions is almost continuous, but not vice versa.

LEMMA 2. Let $K$ be a cardinal number, let $Y$ have weight $\leq k$, and let $f: X \rightarrow Y$ be $a$ arbitrary function. Then the set $A_{C}(f)$ of points of C-almost continuity is K-residua1.

PROOF: $A_{C}(f)=X \backslash_{\tau} \underline{Y}_{K}\left(f^{-1}\left(B_{\tau}\right) \backslash\right.$ Int $D\left(f^{-1}\left(B_{\tau}\right)\right)$, where $B_{\tau}$ 's are elements of the base of $Y$. The sets $f^{-1}\left(B_{\tau}\right) \backslash$ Int $D\left(f^{-1}\left(B_{\tau}\right)\right)$ are of first category by the Banach category theorem.

Recall that a space $X$ is called $k$-Baire if the union of less than or equal to $K$ of nowhere dense subsets of $X$ is a boundary subset of $X$.

COROLLARY 3. Let $K$ be a cardinal number, let $X$ be $K$-Baire, let $Y$ have weight $\leq K$ and let $f: X \rightarrow Y$ be a function. Then there is a dense $G_{K}$ set $A(f)$ such that $f$ is almost continuous at each point of $A(f)$.

Using essentially the fact that. an Infinite metric space of weight $K$ has the cellularity $K$ which is attained: (this means that in metric spaces of weight $K$ there is always a family of open disjoint sets of cardinality $K$ ); and applying similar arguments as in Lin and Lin [2] we have

PROPOSITION 5. Let $\mathrm{Y}$ be a infinite metric space of weight $K$. If $X$ is a space such that every function $f: X \rightarrow Y$ is almost continuous on a dense set $D$ of $X$, then $X$ is $K$-Baire.

We shall now relate some of our results to a we11-known statement in set-theoretical topology. Namely Martin's Axiom says that if $X$ is a CCC compact Hausdorff space, then $x$ is $k$-Baire for each $k<\hat{C}$. This is a topological version of a settheoretical statement.

Somewhat unexpectedly we have the following characterization:

PROPOSITION 6. Martin's Axiom is equivalent to the following statement:

For every compact Hausdorff CCC space $X$, for every cardinal $k<\hat{C}$, for every metric space $Y$ of weight $\leq K$ and for every function $f: X+Y$, there is a dense set of points of almost continuity.

PROOF. The "if" part follows easily from Corollary 3. The "only if" part can be deduced from Proposition 5. 
It appears that the metrizability assumption of $\mathrm{Y}$ in Proposition 5 is essential. However before we demonstrate this fact (Example 8), and further in order to obtain some consequences from the fact that every function $f: X \rightarrow Y$ is almost continuous on a dense set $D \subset \mathbb{R}$, we want to draw the reader's attention to a relationship between the so-called Blumberg property for a function $f$ and $f$ 's property of being almost continuous on a dense subset of its domain.

3. BLUMBERG PROPERTY Recall that a function $\mathrm{f}: \mathrm{X}+\mathrm{Y}$ has the Blumberg property if there is a dense set $D \subset X$ such that the partial function $f \mid D$ is continuous on $D$ in the relative topology.

Further, a space $\mathrm{X}$ is Blumberg iff every function $\mathrm{f}: \mathrm{X} \rightarrow \mathrm{Y}, \mathrm{Y}$ being any second countable has the Blumberg property, see Piotrowsk1 [6] Corollary 2, p. 11.

The following Proposition easily follows from properties of the relative topology.

PROPOSITION 7. (Long and McGehee [7], Theorem 3,p. 177). If $f: X \rightarrow Y$ has the Blumberg property, then $f$ is almost continuous on a dense subset of $X$.

Since there are examples, (e.g. White [8]) of Baire spaces not being Blumberg, the converse of Proposition 7 does not hold in general.

However, in view of Proposition 7, spaces which are suitable for replacement in Theorems $A$ and $B$, the second countable ones, are so-called co-Blumberg spaces.

Recall that a space $\mathrm{Y}$ is said to be co-Blumberg (Brown and Piotrowski [9]) if every function $f: \mathbb{R} \rightarrow Y$ has the Blumberg property.

We shall now show that for $K>\omega$ the metrizability of $Y$ in Proposition 5 is essential. Observe that if $K=\omega$, the metrizability is not necessary, see Theorem $B$.

So we sha11 construct a non $\omega_{1}$-Baire space $X$ and an infinite Hausdorff space $Y$ of weight $\omega_{1}$ such that for any function $f: X \rightarrow Y$ there is a dense set $D$ in $X$ such that $f$ is almost continuous on $D$.

EXAMPLE 8. In fact, let $X$ be any set of cardinality $\omega_{1}$ with the co-finite topology; it is a $\mathrm{T}_{1}$ Baire space which is not a $\omega_{1}$-Baire. Next let $\mathrm{y} \in B N \backslash \mathrm{N}$ be an arbitrary point. Take $Y=\mathbb{N} \cup\{y\}$ as a subspace of $B N$. Clearly, wY $\geq \omega_{1}$. Now, any function $f: X \rightarrow Y$ has a dense set $D$ on which it is almost continuous, see Theorem $A$.

THEOREM 9. Suppose every function $f: R \rightarrow Y$ is almost continuous on a dense subset of $R$. Then $Y$ does not have to satisfy any of the following three conditions:

$$
\begin{aligned}
& \text { (a) } w(\mathrm{Y}) \leq \omega \\
& \text { (b) (under "MA } \left.+\neg \mathrm{CH}^{\prime \prime}\right) \mathrm{c}(\mathrm{Y}) \leq \omega \\
& \text { (c) (under ( } \mathrm{CH}) \text { ) } \mathrm{d}(\mathrm{Y}) \leq \omega
\end{aligned}
$$

PROOF: Ad(a) Y can be either a "co-finite" topology over the reals or an "Arens-Fort" space (see Steen and Seebach [10], Ex - 26). This space is countable Hausdorff; it is not first countable, thus 1 does not satisfy $w(Y) \leq w$. Since $Y$ is a countable, every function $f: R \rightarrow Y$ has the Blumberg property and hence, by Proposition 7 , is almost continuous on a dense subset of $X$.

Ad(b) Let $Y$ be the discrete space of cardinality $\kappa_{1}$. C.learly, $\left.c(Y)\right\rfloor w$. Since MA implies that no open set of the reals is the union of $\kappa_{1}$ or fewer nowhere dense sets, every function $f: R \rightarrow Y$ has the Blumberg property and again by Proposition 7 we are through. 
Ad (c) Assuming ( $\mathrm{CH})$, it was shown in Brown and Piotrowski [9] that the following modification of Ex. 6.3 of Juhasz [11] serves as a space which fails to satisfy $d(Y) \leq w$; however, every function $f: R \rightarrow Y$ has the Blumberg property. In $f$ act, let $Y$ be the set of reals and let $\prec$ denote a well-ordering of $\mathrm{Y}$ which has countable Initial segments. As a base for the topology of $Y$, take sets of the form $U_{x}$, where $U$ is open in the Euclidean topology, $x \in U$, and $U_{x}$ consists of $U$ minus the elements of $U$ which precede $x$ in this ordering. It follows from Proposition 7 that every function $f: R+Y$ is almost continuous on a dense subset of $R$.

REMARK 10. Problem 2 of Lin and Lin [2] p. 185 has been answered in Rose [12], compare Berner [13], see also Byczkowski and Pol [14] where much stronger theorems are proved.

\section{REFERENCES}

1. LIN, Shwu-Yeng T., Almost continuity of mappings, Canad. Math. Bu11. 11(1968), 453-455.

2. LIN, Shwu-Yeng T. and LIN, You-Feng, On almost continuous mappings and Baire spaces, Canad. Math. Bull. 21 (1978), 183-186.

3. TONG, J. C., A note on almost continuous mappings and Baire spaces, Internat. J. Math. \& Math. Sc1. 6(1983), 197-199.

4. TONG, J. C., Another note on almost continuous mappings and Baire spaces, Internat. J. Math. \& Math. Sc1. $7(1984), 619-620$.

5. LUTZER, D. J., On generalized ordered spaces, Dissertationes Math. (Rozprawy Matematyczne) $\underline{89}(1971), 1-36$.

6. PIOTROWSKI, Z, and SZYMANSKI, A., Concerning Blumberg's theorem, Houston J. Math. 10 (1984), 109-115.

7. LONG, Paul E. and MC GEHEE, Earl E., Jr., Properties of almost continuous functions, Proc. Amer. Math. Soc. 24(1970), 175-180.

8. WHITE, H. E., Jr., Topological spaces in which Blumberg's theorem holds, Proc. Amer. Math. Soc. 44(1974), 454-462.

9. BROWN, J. B. and PIOTROWSKI, Z., Co-Blumberg spaces, Proc. Amer. Math. Soc., $96(1986)$, 683-688.

10. STEEN, L. A. and SEEBACH, J. A., Jr., Counterexamples in topology, SpringerVerlag, New York, Heidelberg, Ber11n, 1978.

11. JUHASZ, I., Cardinal Functions in topology, Math. Centre Tracts 34, Amsterdam, 1971 .

12. ROSE, David A., On Levine's decomposition of continuity, Canad. Math. Bull. 21 (1978), 477-481.

13. BERNER, Andrew J., Almost continuous functions with closed graphs, Canad. Math. Bu11. 25 (1982), 428-434.

14. BYCZKOWSKI, T. and POL, R., On the closed graph and open mapping theorems, Bul1. Acad. Polon. Sc1., Ser. Sc1. Math. Astr. Phys. 24 (1976), 723-726. 


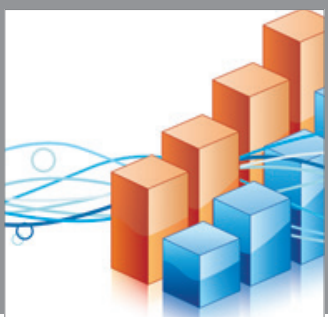

Advances in

Operations Research

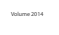

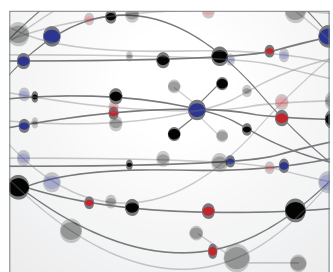

\section{The Scientific} World Journal
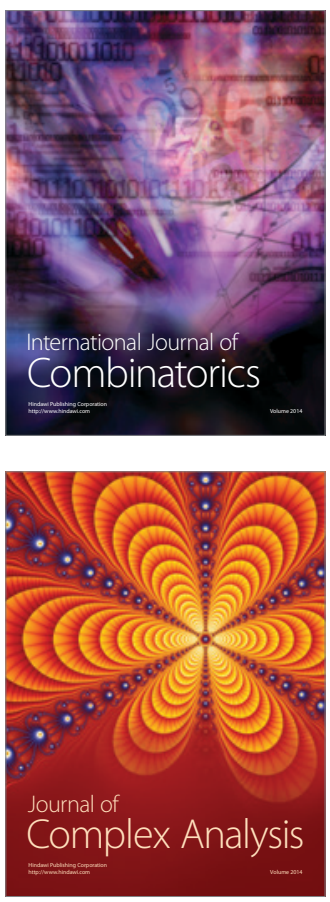

International Journal of

Mathematics and

Mathematical

Sciences
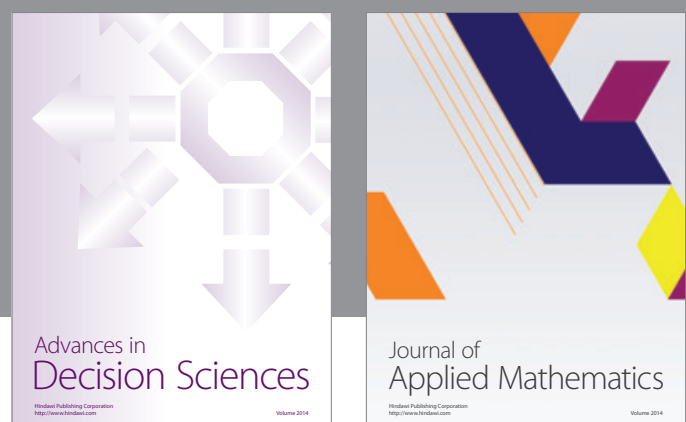

Journal of

Applied Mathematics
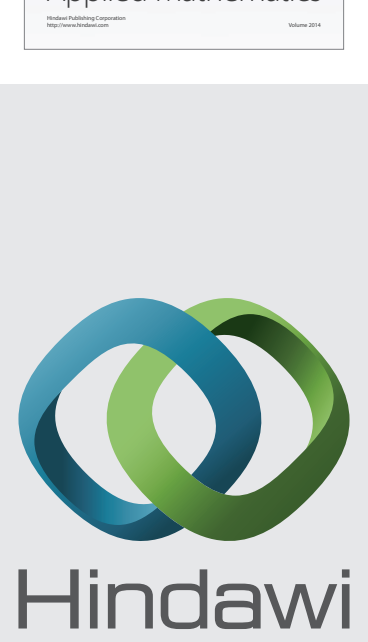

Submit your manuscripts at http://www.hindawi.com
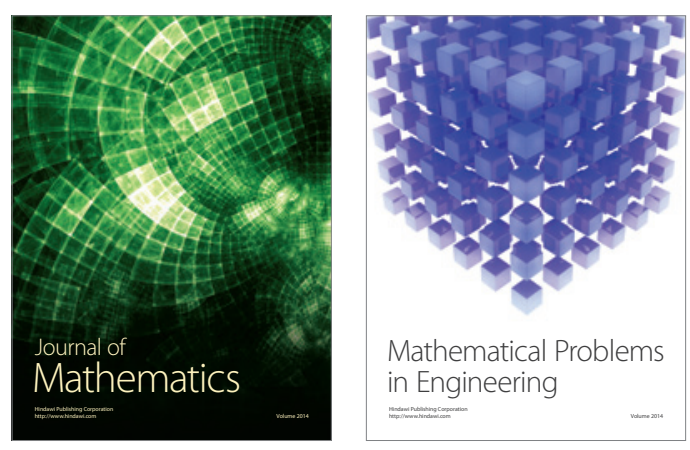

Mathematical Problems in Engineering
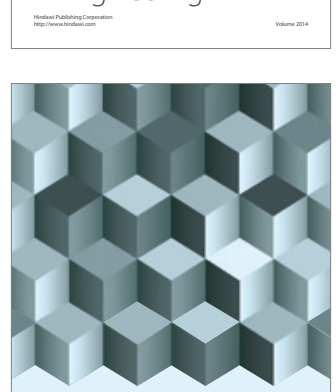

Journal of

Function Spaces
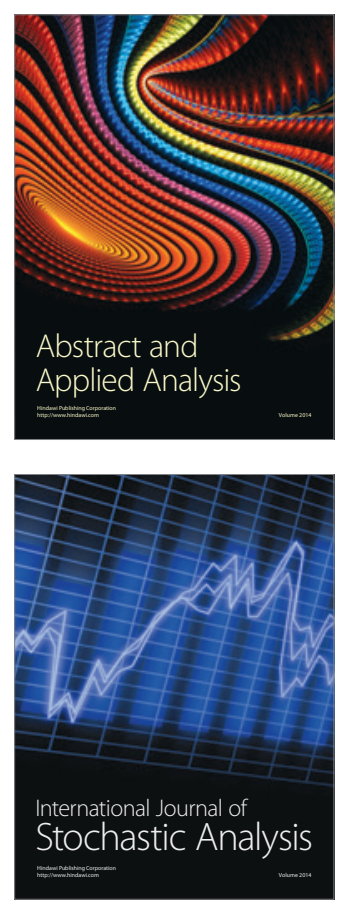

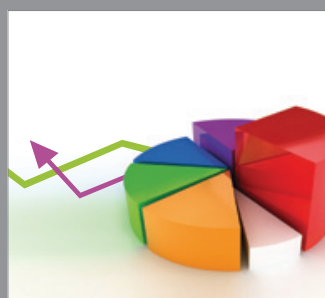

ournal of

Probability and Statistics

Promensencen
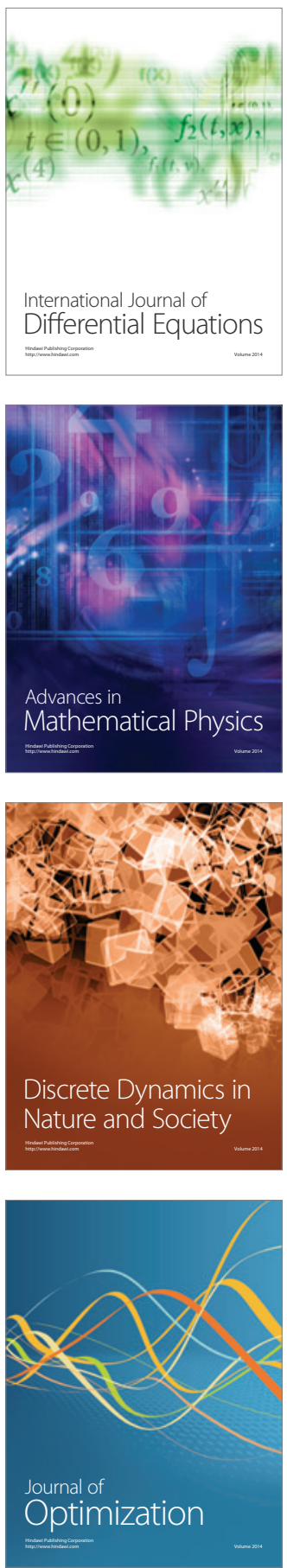